\title{
Medical Informatics
}

\section{Kazuo Maeda*}

Department of Obstrics and Gynecology (Emeritus), Tottori University Medical School, Yonago, Japan

\section{Introduction}

Although the information technology (IT) is broad in medical science, i.e. the medicine itself can be a big IT, computerized managements of information and on-line data transmission will be limitedly discussed in this article.

\section{Electronic patient record}

Data handling: Automatic or key-board input into the personal patient file forming a data-base.

Basic data: name, sex, age, birth date, marriage, family, address, nationality, menstruation; pregnancy; births (women), etc.

\section{Anamnesis: past history}

Present symptoms or history of illness

Laboratory data, ECG, EEG, ultrasound, computer tomography (CT), magnetic resonance imaging (MRI) and other tests

Specific history \& examinations: central nervous system (CNS), eye, otorhinolaryngo- logy, mouth, teeth, respiratory system, heart \& circulation, digestive system, liver, spleen, urogenital system, peritoneum, Obstetrcs \& Gynecology, locomotive system, etc.

Fixed diagnosis:

Treatments:

Surgery:

Radiologic therapy:

Daily, monthly or other chronological changes:

Others:

\section{Central laboratory}

It is an indispensable system for the modern medical science, i.e. every patient sample including the blood, urine, cerebro-spinal fluid etc. taken in the outpatient clinic or in the ward, labeled and sent to the laboratory to receive automated hematological and chemical analysis of samples or pathological examination of tissues even in surgical procedure, and the results are listed on the reporting chart and sent to the attendant physicians making it possible to precisely diagnose the patient state. The reporting chart can be filed and reused in the data base.

\section{Computerized imaging devices}

CT is computerized transparent X-ray tomography. MRI is analyzed under high magnetic field to visualize computerized structure images. Three and four dimensional (3D and 4D) ultrasound are computerized $\mathrm{B}$-mode ultrasound imaging. CT and MRI are indispensable to analyze the detailed anatomical CNS structure and arterial vessels. The 3D CT visualizes bone structure. The MRI does not need to take care of X-ray radiation. Rapid MRI shows fetal movements. The $3 \mathrm{D}$ ultrasound shows fetal face and $4 \mathrm{D}$ one analyzes fetal face expression and fetal behavior by fetal movements. Every image is stored in computerized data bases. Whole elementary $3 \mathrm{D}$ ultrasound voxel data are on-line transmitted to an expert for the reconstruction of 3D images in the computer for the remote diagnosis.

\section{The use of patient record in the other department or operation theater}

The same electronic record as the first department is used in the other department of the same hospital, with the same patient ID number. The data base and the medical images of the patient are displayed in the large flat panel in the operation theater, but the chart record written on the paper and films of X-ray, MRI or ultrasound photo do not enter into the theater in order to avoid bacterial pollution, i.e. only computerized patient information is allowed to enter the operation theater.

The transport of patient record in the visit to the other hospital

The electronic summary and any necessary data should be transferred to the other hospital before the visit of refered patient to receive a particular medical examination, but not at the same time of the visit, or to attach the introduction letter to the patient, i.e. the details of patient status should be informed to the introducing hospital prior to the patients arrival, in order to receive the examination immediately after arriving at the hospital. The stay in the hospital is saved by the information prior to the visit, and the patient will come back original hospital soon. The results of special examination is also sent back original hospital by an internet communication. Every patient data are guarded by the internet security.

\section{Centralized automated computer monitoring of fetuses in the birth}

Fetal monitoring with the visual diagnosis of the chart record (cardiotocogram, CTG) of continuous fetal heart rate (FHR) and uterine contraction is time consuming and troublesome duty of obstetrician or the staff. Computerized FHR analysis and automatic warning of abnormal FHR will solve the problem, particularly in the hospital handling many births. The FHR and uterine contraction signals are sent to the center by local area network or radio-wave telemetry and received by the time sharing system to enable to simultaneously analyze 100 feal data. The computer system was programmed by the FHR score calculation enabling to detect fetal asphyxia in early stage of labor, abnormal FHR changes, pathological outcome probability detected by neural network system, and FHR frequency analysis to differentiate sinusoidal FHR and physiological change and to estimate the loss of

${ }^{*}$ Corresponding author: Kazuo Maeda, Department of Obstrics and Gynecology (Emeritus), Tottori University Medical School, Yonago, Japan, E-mail: maedak@mocha.ocn.ne.jp

Received August 21, 2013; Accepted August 22, 2013; Published August 26 , 2013

Citation: Maeda K (2013) Medical Informatics. J Health Med Informat 4: e109. doi:10.4172/2157-7420.1000e109

Copyright: (c) 2013 Maeda K. This is an open-access article distributed under the terms of the Creative Commons Attribution License, which permits unrestricted use, distribution, and reproduction in any medium, provided the original author and source are credited. 


\section{DETAILS OF THE CENTRALIZED AUTOMATIC MONITORING}

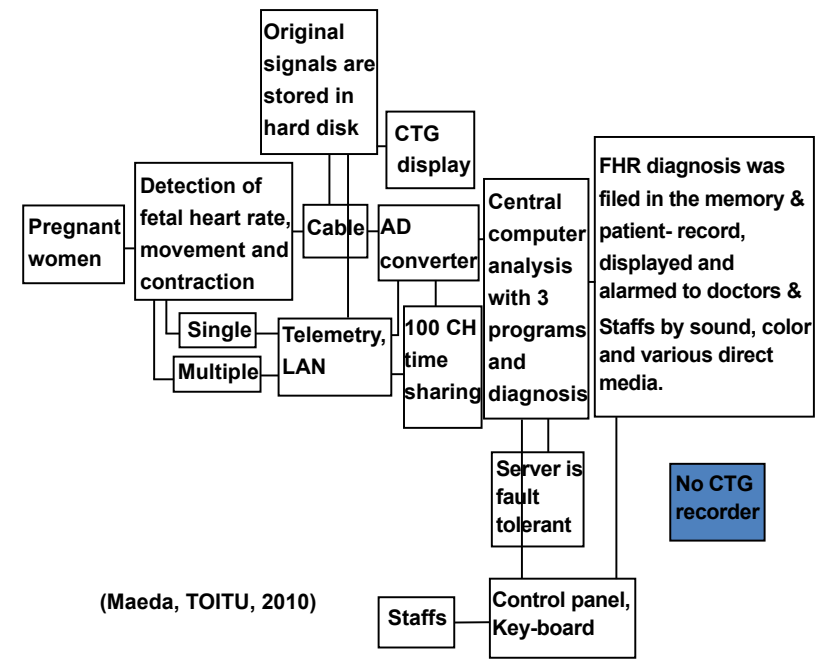

Figure 1: Central computerized automatic fetal heart rate monitoring system [1].

FHR variability. The abnormal FHR and potential pathologic prognosis are immediately and directly alarmed to the attending physician (Figure 1), [1]. Such monitoring

\section{Laparoscopic surgery assisted by the robot computer}

The laparoscopic surgery is less invasive to the patient than the surgery under laparotomy and recommended to various intraperitoneal surgeries. Delicate movement of mechanical manipulator is enlarged and shown to the operator by the assistance of Da Vinci computer in the surgery, enhancing the smooth surgical technique under laparoscope. Therefore, robot computer assisted laparoscopic surgery is widely applied in pelvic surgery.

\section{Exchange of medical information through internet groups}

Since it was needed to exchange medical experiences, news in medical field, and various opinions, it was achieved in some internet medical groups discussing active news, experiences and opinions, despite the congress of a computer society gathering the people in a place through long trips is postponed, because the exchange of opinion through internet is more convenient than the congress presentation.

\section{On-line, open-access publication of medical reports}

It is common now to publish a scientific paper by on-line and open access journal, instead to publish the paper in a printed manner, enhancing the acquisition of new information in medical field, instead to purchase many journals or requesting reprints to authors.

\section{Web-site medical libraries}

It is common to read abstracts of recent as well as previous works reported in medical journals searched by the working subjects, authors names and years in various on-line medical libraries. They promotes to study previous works in the investigation of new subjects and in the writing of a medical report

\section{References}

1. Kazuo Maeda, Masaji Utsu, Yasuaki Noguchi, Fujihiko Matsumoto, Takashi Nagasawa (2012) Central computerized automatic fetal heart rate diagnosis with a rapid and direct alarm system. The Open Medical Devices Journal 4 : 28-33. 